\title{
A High Voltage RF Oscillator for Driving Multipole Ion Guides
}

\author{
Peter B. O'Connor and Catherine E. Costello \\ Boston University School of Medicine Mass Spectrometry Resource, Boston, Massachusetts
}

William E. Earle

Boston University Electronics Design Facility, Boston, Massachusetts

\begin{abstract}
A high voltage RF oscillator circuit has been designed and constructed for driving multipole ion guides. The circuit is tunable from $500 \mathrm{kHz}$ to $1.5 \mathrm{MHz}$ by changing a capacitor and provides $0-1000 \mathrm{~V}_{p-p}$ that is controlled by a $0-10 \mathrm{~V}$ input using a negative feedback circuit. This inexpensive circuit uses a set of high voltage transistors oscillating in tandem and does not require tuning of the resonance drive frequency as the oscillator automatically resonates at the (LC) $)^{-1 / 2}$ frequency. Matrix-assisted laser desorption/ionization-Fourier transform mass spectrometry (MALDI-FTMS) mass spectra were acquired using this tunable RF oscillator circuit to allow transmission of protein ions in the 8.5-39 $\mathrm{kDa}$ range through the quadrupole ion guide from the ion source to the mass analyzer. (J Am Soc Mass Spectrom 2002, 13, 1370-1375) (C) 2002 American Society for Mass Spectrometry
\end{abstract}

$\mathrm{M}$ ultipole ion guides [1] are routinely used throughout mass spectrometry on a wide variety of instruments. These ion guides efficiently transport ions from one location to another and perform particularly well at higher pressures so that they are used to focus ions past pumping restrictions or to trap ions for control of ion kinetic and vibrational energy. The high voltage RF needed for the ion guides is normally generated by an LC oscillating circuit (called a "tank circuit") which is in turn driven by a high power amplifier. Several groups have reported extending the mass range of commercial instruments by changing the coil of the LC circuit to adjust the oscillating frequency [2-4]. The theory of multipole ion guides is well described in a review article by Gerlich [1], with the net effect for mass ranges used in biomolecular mass spectrometry that a frequency range of $0.3-1.5 \mathrm{MHz}$ and a voltage range of $<2 \mathrm{kV}_{p-p}$ are typically used.

Commercial instruments come equipped with circuitry for driving the ion guides, but for home built systems, it is necessary to build or purchase these electronics separately. While they can be purchased commercially, the cost ( $\$ 8 \mathrm{k}-\$ 20 \mathrm{k}$ each) is often prohibitive, particularly when the instrument will require several sets of these electronics, as is the case with the quadrupole-FTMS [5, 6] and quadrupole-TOF instruments $[7,8]$. Additionally, these commercial circuits are usually tuned to one frequency, which can be adjusted

Published online October 24, 2002

Address reprint requests to Dr. P. B. O'Connor, Department of Biochemistry Mass Spectrometry Resource, Boston University School of Medicine, 715 Albany Street R806, Boston, MA 02118. E-mail: poconnor@bu.edu only by changing the coil and then reoptimizing the drive frequency. The circuit we present here is a low cost high voltage oscillator whose resonance frequency automatically adjusts to the resonance frequency of the tank, and a negative feedback automatic gain control (AGC) circuit is used to control the amplitude of the output. Changing the frequency only requires changing a capacitor, and a $0-10 \mathrm{~V}$ DAC controls the amplitude of the output. It can be used to control all RF-only multipole ion guides including quadrupoles, octopoles, and hexapoles, and RF-DC capability can be added easily.

This article describes an oscillator design that modifies a published design [9] to use transistors rather than vacuum tubes for reduced cost, size, and circuit simplicity. The circuit is also modified to use a tightly coupled transformer for separating the DC offset of the tank circuit from the power supply voltage of the transistors. The design provides a controlled amplitude RF sine-wave output across a floating LC tank circuit over a frequency range of $500 \mathrm{kHz}$ to $1.5 \mathrm{MHz}$ with a peak-to-peak voltage of up to $1000 \mathrm{~V}_{p-p}$.

\section{Circuit Design}

Figure 1 shows the basic circuit for the oscillator, and Figure 2 shows the full circuit design with the details discussed below. All circuit diagrams are drawn so that connections are indicated by either a line between two distinct points or from a point to a "T" connection; a cross over is not a connection unless specifically noted. The tank circuit is electrically isolated from the transistor oscillator so that it does not need any high-voltage 


\section{To multipole ion guide}

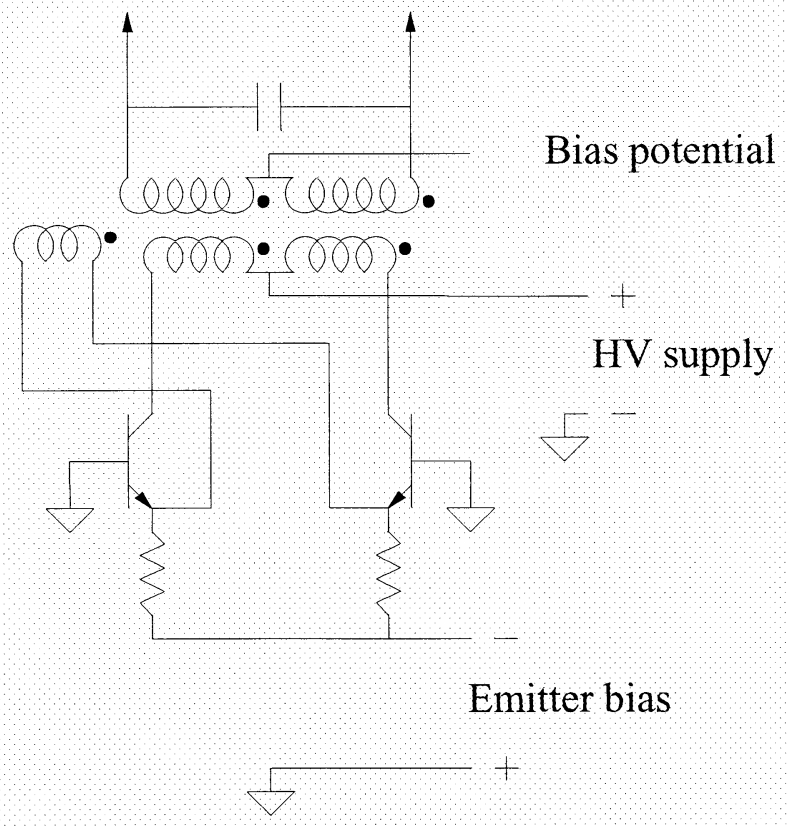

Figure 1. The basic common-base transistor oscillator circuit coupled to a traditional LC tank circuit on the multipole rods.

isolating capacitors to remove the DC voltage used by the transistors. A center tap on the secondary coil of the transformer allows DC biasing of the multipoles. The transistors are operated in a common-base configuration in order to preserve as much of the transistor bandwidth as possible, whereas the more usual common-emitter configuration would normally reduce the

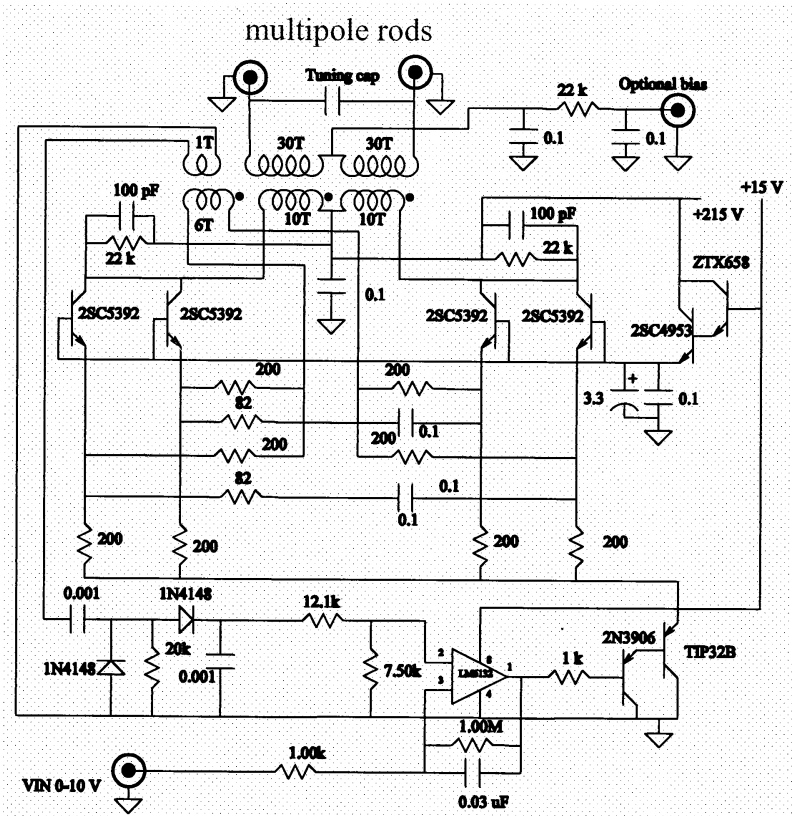

Figure 2. The complete diagram for the oscillator and automatic gain control (AGC) feedback circuit. Unless otherwise noted, resistance values are in ohms, and capacitance values are in microfarads. bandwidth. The transistor selected for use is the Panasonic 2SC5392 (Digikey, Thief River Falls, MN), rated to have a $3 \mathrm{~dB}$ cutoff frequency of $20 \mathrm{MHz}$ in the commonbase configuration.

Feedback required to achieve oscillation comes from a small feedback coil and pair of $200 \Omega$ series resistors connected between the transistor emitters. The series resistor is needed to convert the feedback voltage into a feedback current. The amplitude of the oscillation is set by the DC value of the emitter current, which is determined by the bias voltage to the emitter resistors. The feedback signal is $180^{\circ}$ out of phase with the oscillator so that, once oscillation begins, the amplitude of oscillation increases until the emitter current to the emitter receiving the positive voltage swing is reduced to zero. Since the two emitters are in series with the feedback coil, the cutoff of emitter current to one transistor also serves to limit the current to the other one, preventing a further increase in oscillation amplitude. The reader will note that this limiting mechanism does not depend on saturation of the collector voltage, which would significantly alter the transistor characteristics and probably shift the oscillation frequency away from that of the tank circuit. Small signal transistor oscillators often do depend on transistor saturation to limit oscillation amplitude, but this is only satisfactory when transistors being used exhibit negligible side effects due to saturation.

The RF amplitude is controlled by adjustment of the emitter current. When the negative bias voltage to the emitters is increased, the feedback amplitude required to cut off emitter current will also increase, allowing the oscillation amplitude to increase. Thus, the voltage developed across the tank circuit is proportional to the emitter bias voltage.

\section{Coil Design}

Devising an acceptable coil design was a major part of this oscillator project. Many coils were wound before settling on the present design. To maintain as high a $Q$ (meaning low loss) as possible, relatively large wire sizes were used; the tank circuit is wound from 18gauge (1.024 mm diameter) copper magnet wire and the transistor drive coil and feedback coil use 16-gauge copper magnet wire $(1.291 \mathrm{~mm}$ diameter).

A high coupling constant between the primary and the secondary coil of the transformer is crucial. Without it, the transistors, which are hooked to the primary coil, will tend to oscillate at a frequency other than that set by the inductance (provided by the secondary coil) and the capacitance of the tank circuit. It was found to be difficult to adequately couple the transistor winding to a relatively long center-tapped tank coil because each transistor tended to drive only its own half of the tank coil rather than the entire coil. Splitting the secondary coil into two identical windings, one on top of the other, with the primary coil on top of both, proved to give the best coupling. The use of overlapping tank coils also 
reduces the required number of turns in the tank coil because of the increased inductance of overlapping coils.

The final coil design uses a $5.08 \mathrm{~cm}$ diameter poly(vinyl chloride) PVC coil form with each half of the secondary tank coil consisting of 30 turns of 18-gauge magnet wire. The primary transistor winding consists of 20 turns of 16-gauge magnet wire, center-tapped, essentially completely covering the tank coil windings. Three layers of 0.004 inch $(0.1 \mathrm{~mm})$ thick Mylar (DuPont, Wilmington, DE) serve to insulate the tank coil halves from each other and the transistor coil from the outer tank coil half. The feedback coil consists of 6 turns of 16-gauge magnet inside the coil form, with the turns spread to equal the length of the other coils. There is also a single turn sensing loop used by the feedback (AGC) circuit; this is merely a single loop of insulated hookup wire placed over the center of the other windings.

\section{Transistors and Power Dissipation}

The experimentally determined full power requirements at maximum $\left(1000 \mathrm{~V}_{p-p}\right)$ amplitude is about $40 \mathrm{~W}$ at $500 \mathrm{kHz}$ and $20 \mathrm{~W}$ at $1.5 \mathrm{MHz}$, with half the power being dissipated in the tank circuit and the other half in the transistors. The transistor chosen for the oscillator is the Panasonic 2SC5392. This is a $500 \mathrm{~V} 20 \mathrm{MHz}$ transistor in a TO-220 case with a $25 \mathrm{~W}$ dissipation at a $25{ }^{\circ} \mathrm{C}$ case temperature. However, actual dissipation is determined by thermal design so that, with a good heat sink and fan, $10 \mathrm{~W}$ of heat dissipation would be considered a reasonably conservative estimate. Since the two oscillator transistors would have to dissipate up to $20 \mathrm{~W}$ (10 $\mathrm{W}$ each), it was decided to use parallel transistors, four total, to limit the dissipation of each transistor to $5 \mathrm{~W}$. This is a sufficient derating to allow continuous operation at $20 \mathrm{~W}$ with a minimum chance of premature transistor failure.

This transistor also has a relatively low collector-base capacitance for a power transistor of about $100 \mathrm{pF}$. Because of the 3:1 turns ratio between the tank coil and the driver coil, this capacitance will effectively be only $11 \mathrm{pF}$ across half the tank coil, thereby not significantly influencing the tuning of the tank circuit. However, connecting the pairs of transistors in parallel, as is discussed above, also doubles the effective transistor capacitance seen by the tank coil to $22 \mathrm{pF}$, but this value is still acceptable.

The 3:1 turns ratio between tank coil and driver coil means that with the maximum $1000 \mathrm{~V}_{p-p}$ across the tank coil, only $333 \mathrm{~V}_{p-p}$ will appear across each transistor. With a transistor supply voltage of $215 \mathrm{~V}$, the collector will swing from 49 to $381 \mathrm{~V}$. Thus, collector saturation is avoided.

\section{Emitter Feedback}

Due primarily to the decision to use parallel transistors, the coupling of the feedback coil to the emitters in-

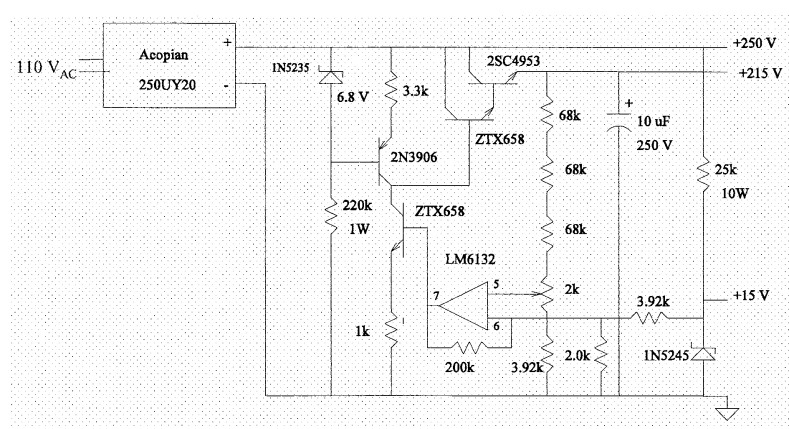

Figure 3. The power supply for the oscillator. Unless otherwise noted, resistance values are in ohms, and capacitance values are in microfarads.

volves using multiple resistors and decoupling capacitors to guarantee an equal power distribution among the four transistors, as seen in Figure 2. The reader will also note also that there are two additional resistors that effectively connect the two emitter pairs. These resistors serve to limit the amplitude of the reverse emitter-base voltage when the oscillation amplitude is limiting. Before limiting occurs, the emitter-emitter impedance is very low so these resistors have almost no effect on circuit operation. However, when limiting begins, the impedance of the transistor that is cut off becomes rather high and might allow the reverse emitter-base voltage to exceed the maximum rating of the transistor, were it not for the shunting effect of these resistors.

\section{Automatic Gain Control}

AGC is provided by comparing the rectified signal from the sensor loop with the desired signal using a National LM6134 op-amp (Digikey, Thief River Falls, MN) so that a $0-10 \mathrm{~V}_{\mathrm{dc}}$ will linearly correlate with a $0-1000 \mathrm{~V}_{p-p}$ output. The difference between the measured and desired signals is amplified by the op-amp and used to drive a Darlington-connected transistor pair to provide the emitter bias for the oscillator transistors. The gain and frequency response of the amplifier was chosen to guarantee a stable AGC circuit.

\section{Power Supply}

The supply voltage used by the transistors of the oscillator is $215 \mathrm{~V}, 200 \mathrm{~V}$ between the collectors and bases and $15 \mathrm{~V}$ to power the op-amp and Darlington driver. It might be possible to use an unregulated supply and depend upon the AGC circuit to maintain the proper oscillation amplitude in the presence of a varying supply voltage, but the cost and complexity of regulating the supply voltage is minimal, so a regulator is included as a part of the design. Figure 3 shows the power supply diagram. 


\section{Passive Components}

All resistors were 1\%, 1/4 W metal film resistors, and all capacitors were low accuracy ceramic capacitors except for the output tuning capacitors which were high quality silvered mica capacitors as noted below.

\section{Oscillator Tuning}

With the specified coil design, the external capacitance needed across the tank coil is $680 \mathrm{pF}$ at $500 \mathrm{kHz}$ and 45 $\mathrm{pF}$ at $1.5 \mathrm{MHz}$. This capacitance is set by the sum of the tuning capacitor shown in Figure 2 and the capacitance of the multipole. Silvered mica capacitors were chosen because of their low loss and high stability. Since the tank circuit can develop up to $1000 \mathrm{~V}_{p-p}$, the capacitors require a rating of at least $1000 \mathrm{~V}$. While many mica capacitors are only rated for $500 \mathrm{~V}$, these can still be used if two capacitors having equal values are placed in series so that each capacitor only sees $500 \mathrm{~V}$. Since the capacitor needed for tuning the circuit is in parallel with the multipole, the actual capacitance value needed for a given frequency is the sum of the multipole and tuning capacitance. In the data shown below, the multipole capacitance for the $\sim 1 \mathrm{~m}$ long RF-only quadrupole (6.35 $\mathrm{mm}$ diameter cylindrical rods with pole-to-pole spacing of $5.5 \mathrm{~mm}$ mounted inside a $\sim 35.5 \mathrm{~mm}$ i.d. stainless steel support cylinder) is $\sim 162 \mathrm{pf}$ (including $\sim 40 \mathrm{~cm}$ MHV cable).

\section{Cooling and Enclosure}

The four oscillator transistors were mounted to heat sinks and placed in the airflow of a cooling fan in order to achieve the heat dissipation levels discussed above. The power supply regulator transistor and the power Darlington transistor also needed heat sinks and need to be in the air flow. Furthermore, the entire oscillator was placed inside a metal enclosure in order to minimize RF radiation, since the oscillation frequencies fall entirely within the AM broadcast band.

\section{One Subtlety}

The only known subtlety of the design is that the oscillator transistors tend to oscillate at a very high frequency $(40 \mathrm{MHz})$ unless the collectors are bypassed to the $215 \mathrm{~V}$ supply by small capacitors $(100 \mathrm{pF})$. These capacitors do not significantly lower the normal oscillation frequency but they do reduce the effective bandwidth of the transistors, thereby eliminating the unwanted high-frequency oscillation.

\section{Methods}

The instrument modified to test this circuit is the high pressure MALDI FTMS previously described [10, 11]. This instrument uses an octopole ion guide as an ion trap for accumulation of MALDI ions and a quadrupole

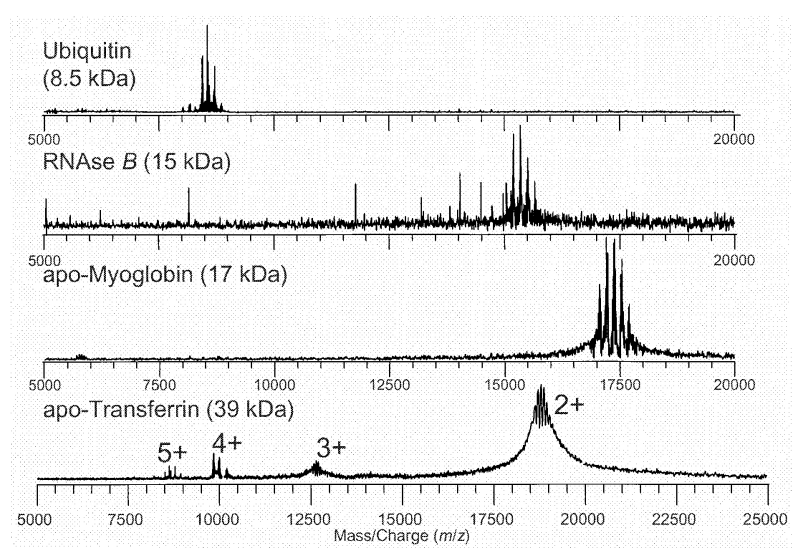

Figure 4. MALDI-FTMS spectra of 8.5 to $39 \mathrm{kDa}$ proteins and glycoproteins displayed in the $m / z 5000-25,000$ range.

ion guide to transfer ions from the source to the ICR cell. Samples for UV (337 $\mathrm{nm})$ MALDI were prepared using the "dried droplet" method [12]. The matrix was a saturated solution of 2,5 dihydroxybenzoic acid (DHB) [13] in acetone. Proteins were purchased from Sigma (St. Louis, MO), except for the N-Lobe of apomyoglobin which was a generous gift from Professor Kaltashov [14]. For the spectra shown here, typically $\sim 1$ pmol of sample was deposited along with $\sim 1 \mu \mathrm{L}$ of matrix solution. Standard ICR conditions were used, except that the frequency range of the excite/detect was shifted to accommodate the high $\mathrm{m} / \mathrm{z}$, and the amplitude had to be reoptimized to $\sim 150 \mathrm{~V}_{p-p}$.

The instrument is controlled by the IonSpec (Irvine, CA) Explorer data system version 6.72. The RF amplitude is set using one of the eight additional 12 bit + /$10 \mathrm{~V}$ DACs provided by the system. During the ion transfer event, the voltage is set to a variable amplitude from $\sim 0.50$ to $10 \mathrm{~V}$ corresponding to an output of $50-1000 \mathrm{~V}_{p-p}$. The RF amplitude is shut off by setting the DAC input to 0 volts, but in practice, due to a fear of residual low amplitude oscillation causing noise spikes in the spectra, a value of $-0.25 \mathrm{~V}$ is used. The two multipoles used are a quadrupole (rod diameter $=6.35$ $\mathrm{mm}$, pole to pole distance $=5.5 \mathrm{~mm}, 162 \mathrm{pf}$ ) and an octopole (rod diameter $=6.35 \mathrm{~mm}$, pole to pole distance $=12.7 \mathrm{~mm}, 32 \mathrm{pf})$. The quadrupole is driven by the new design at $\sim 580 \mathrm{kHz}$ and $\sim 800 \mathrm{~V}_{p-p}$, the octopole is driven by a signal generator (Wavetek model 19, Fluke Corporation, Everett, WA), a high voltage amplifier (ENI model 2100L, Rochester NY), and a home built LC tank circuit operated at $\sim 680 \mathrm{kHz}$ and $\sim 1.5 \mathrm{kV}_{p-p}$.

\section{Results and Discussion}

Figure 4 shows the regions containing abundant ion signals in the mass spectra of a series of proteins and glycoproteins ranging in molecular weight from 8.5 to $39 \mathrm{kDa}$ that were ionized using the high pressure MALDI source. Clearly the ions from $8.5 \mathrm{kDa}(1+)$ to 39 $\mathrm{kDa}(2+)$ are generated, trapped, and transferred to the cell in a single experiment. Additionally, the apotrans- 


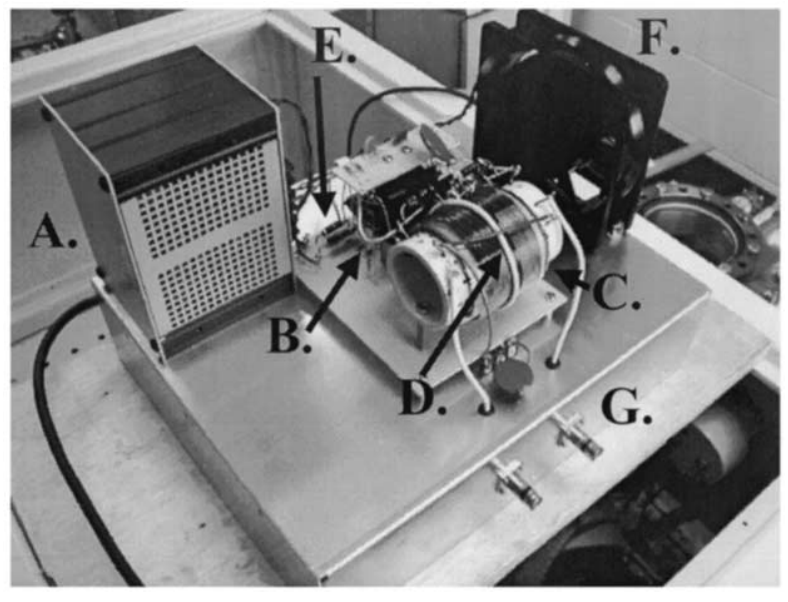

Figure 5. Annotated photo of the oscillator. (A) power supply; (B) transistors with heat sinks; (C) main coil; (D) single loop feedback coil; (E) rectifier/comparator circuit; (F) fan; (G) MHV outputs for multipoles.

ferrin spectrum included a low abundance (signal/ noise $\sim 2$ ) peak that corresponded to the singly charged ion whose low abundance was likely due to the fact that the RF amplitude and frequency had not been optimized for transmission of $m / z \sim 40,000$. Extending ion transport capability to the $m / z 40,000$ range will require modification of the circuit to decrease the frequency or increase the amplitude as discussed below. There were no noise spikes from the RF oscillator circuit (although several from the turbopumps were apparent) indicating that it does indeed turn completely off during detection.

Figure 5 is an annotated photograph of the circuit. Note that the coil and oscillator occupy an approximately $10 \mathrm{~cm}$ cube, not including the power supply and fan. The circuit is mounted in an aluminum enclosure to avoid additional RF interference as the frequencies of interest are generally within the AM radio band. The top of the enclosure (not shown) is formed from sheet metal and copper mesh to allow adequate airflow, the base (shown) is an aluminum box. The tuning capacitor is not visible, but is mounted directly on the back of the MHV connectors ( $\mathrm{G}$ in Figure 5). This circuit modifies an existing vacuum tube based design [9] to use transistors and in favor of a transformer based tank circuit rather than using blocking capacitors, but there is a tradeoff that must be made between the two circuit designs. While transistors are smaller, cheaper, and easier to acquire and work with, vacuum tubes can more readily handle high voltage and high power operation. Additionally, vacuum tubes can be run hot, while the transistors require substantial heat fins and a cooling fan. This circuit does not provide a clear gain in performance compared to a vacuum tube based design, but it provides substantial advantages in that it is small, inexpensive, and relatively simple to build and operate.

Some commercial RF quadrupole power supplies can generate much higher (e.g., $5 \mathrm{kV}_{p-p}$ ) $\mathrm{RF}$ amplitudes compared to the $1 \mathrm{kV}_{p-p}$ available here. The maximum amplitude needed is defined by the inscribed radius of the multipole, the pole number, the maximum $\mathrm{m} / \mathrm{z}$ transmission required and the frequency of the multipole. In cases of very high $\mathrm{m} / \mathrm{z}$ operation (e.g., $>25000$ $\mathrm{m} / \mathrm{z}$ ) it may be desirable to use large inscribed radius and high amplitude, but often decreasing the frequency will also work $[2,3]$. The $1 \mathrm{kV}_{p-p}$ maximum amplitude of this circuit is defined by the 3:1 turn ratio on the transformer and can be adjusted by changing the coil, but the effect on this change of turn ratio is likely to be a decrease in the frequency tuning range of the output. Additionally, it is easy to add DC offset capability to this circuit for operation in RF-DC mass filtering mode using standard capacitive coupling techniques. Doing so simply requires addition of a set of blocking capacitors to the output and an optoisolator chip such as the Burr Brown ISO100 (Digikey, Thief River Falls, MN). This circuit is designed for single frequency mass filtering on a $+/-1 \%$ mass $(+/-10 \mathrm{Da}$ at $1 \mathrm{kDa})$ level and is perfectly adequate for RF-only mode mass transport or trapping. However, as the amplitude stability and $\mathrm{RF} / \mathrm{DC}$ ratio stability is not guaranteed at the $0.1 \%$ level or lower, this circuit is likely to be of limited utility for mass scanning (or narrow band mass isolation) as the instability of these crucial parameters would induce mass calibration errors.

In order to reach even higher $\mathrm{m} / \mathrm{z}$ ranges, the circuit will have to be modified to reduce the frequency and/or increase the amplitude. Shifting the frequency range of the circuit will require, primarily, adjustment of the coil design. Increasing the amplitude will require either finding a transistor capable of operation at higher voltage, or changing the $3: 1$ turn ratio of the transformer for a higher ratio. While the latter change is simple in concept, care will have to be taken that the oscillator is still well coupled to the tank.

In some cases, one RF driver that is capacitively coupled could drive multiple RF ion guides provided that it can handle the additional capacitive load. This is commonly done with commercial instruments and is not difficult, but it does introduce a certain degree of inflexibility in the instrumentation that can become limiting in home-built instruments, which are constantly being modified. If the RF driver circuitry is sufficiently inexpensive, the limitations of capacitive coupling can be avoided by simply duplicating the electronics.

\section{Conclusions}

A high voltage RF oscillator circuit is presented for control of various types of multipole ion guides. Tuning the frequency and amplitude of this circuit is trivial. Amplitude is controlled by a $0-10 \mathrm{~V}_{\mathrm{dc}}$ DAC from the data system, and frequency is controlled by changing the tuning capacitor. The use of an oscillator design like this means that the frequency never needs to be adjusted as the circuit automatically oscillates at the $(\mathrm{LC})^{-1 / 2}$ resonance frequency. The flexibility of this 
circuit allows exploration of a wide parameter space for optimal tuning of mass transmission capabilities of a multipole ion guide.

Additionally, while the circuit was fairly expensive to develop (although still totaling less than a commercial system), it is remarkably inexpensive to build, costing $<\$ 200$ in parts, with the biggest expense being the power supply. At this time, circuitry is being added to allow a DC offset between the quadrupole rods for $\mathrm{RF} / \mathrm{DC}$ operation, and a printed circuit board is being generated that will decrease the assembly cost still further. Future use of this circuit in the BUSM Mass Spectrometry Resource is projected to be $\sim 6$ of these circuits for the FTMS alone and additional units would be suitable for modification of the quadrupole orthogonal time-of-flight instruments. Thus, the cost savings compared to purchasing full commercial multipole drivers is significant.

\section{Acknowledgments}

The authors thank Mark McComb of the BUSM Mass Spectrometry Resource and Eric Hazen of the Boston University Electronics Design Facility for helpful discussions. The work was supported by NIH P41-RR10888.

\section{References}

1. Gerlich, D. In State-Selected and State-to-State Ion-Molecule Reaction Dynamics; Ng, C.-Y.; Baer, M., Eds.; John Wiley and Sons, Inc.: New York, 1992; Vol. LXXXII, pp. 1-176.

2. Collings, B. A.; Douglas, D. J. An Extended Mass Range Quadrupole for Electrospray Mass Spectrometry. Int. J. Mass Spectrom. Ion Processes 1997, 162, 121-127.

3. Winger, B. E.; Light-Wahl, K. J.; Ogorzalek Loo, R. R.; Udseth, H. R.; Smith, R. D. Observation and Implications of High Mass-to-Charge Ratio Ions from Electrospray Ionization Mass Spectrometry. J. Am. Soc. Mass Spectrom. 1993, 4, 536-545.

4. Sobott, F.; Hernandez, H.; McCammon, M. G.; Tito, M. A.; Robinson, C. V. A Tandem Mass Spectrometer for Improved Transmission and Analysis of Large Macromolecular Assemblies. Anal. Chem. 2002, 74, 1402-1407.
5. Senko, M. W.; Hendrickson, C. L.; Emmett, M. R.; Shi, S. D. H.; Marshall, A. G. External Accumulation of Ions for Enhanced Electrospray Ionization Fourier Transform Ion Cyclotron Resonance Mass Spectrometry. J. Am. Soc. Mass Spectrom. 1997, 8, 970-976.

6. Meng, F.; Cargile, B. J.; Patrie, S. M.; Johnson, J. R.; McLoughlin, S. M.; Kelleher, N. L. Processing Complex Mixtures of Intact Proteins for Direct Analysis by Mass Spectrometry. Anal. Chem. 2002, 74, 2923-2929.

7. Morris, H. R.; Paxton, T.; Dell, A.; Langhorne, J.; Berg, M.; Bordoli, R. S.; Hoyes, J.; Bateman, R. H. High Sensitivity Collisionally-Activated Decomposition Tandem Mass Spectrometry on a Novel Quadrupole/Orthogonal-Acceleration Time-of-Flight Mass Spectrometer. Rapid Commun. Mass Spectrom. 1996, 10, 889-896.

8. Chernushevich, I. V.; Ens, W.; Standing, K. G. OrthogonalInjection TOF-MS for Analyzing Biomolecules. Anal. Chem. 1999, 71, 452A-461A.

9. Jones, R. M.; Gerlich, D.; Anderson, S. L. Simple RadioFrequency Power Source for Ion Guides and Ion Traps. Rev. Sci. Inst. 1997, 68, 3357-3362.

10. O'Connor, P. B.; Costello, C. E. A High Pressure MatrixAssisted Laser Desorption/Ionization Fourier Transform Mass Spectrometry Ion Source for Thermal Stabilization of Labile Biomolecules. Rapid Commun. Mass Spectrom. 2001, 15, 1862-1868.

11. O'Connor, P. B.; Mirgorodskaya, E.; Costello, C. E. High Pressure Matrix-Assisted Laser Desorption/Ionization Fourier Transform Mass Spectrometry for Minimization of Ganglioside Fragmentation. J. Am. Soc. Mass Spectrom. 2002, 13, 402-407.

12. Kussmann, M.; Nordhoff, E.; Rahbeknielsen, H.; Haebel, S.; Rossellarsen, M.; Jakobsen, L.; Gobom, J.; Mirgorodskaya, E.; Krollkristensen, A.; Palm, L.; Roepstorff, P. Matrix-Assisted Laser Desorption/Ionization Mass Spectrometry Sample Preparation Techniques Designed for Various Peptide and Protein Analytes. J. Mass Spectrom. 1997, 32, 593-601.

13. Strupat, K.; Karas, M.; Hillenkamp, F. 2,5-Dihydroxybenzoic Acid: A New Matrix for Laser Desorption-Ionization Mass Spectrometry. Int. J. Mass Spectrom. Ion Processes 1991, 111, 89-102.

14. Eyles, S. J.; Dresch, T.; Gierasch, L. M.; Kaltashov, I. A. Unfolding Dynamics of a Beta-Sheet Protein Studied by Mass Spectrometry. J. Mass Spectrom. 1999, 34, 1289-1295. 